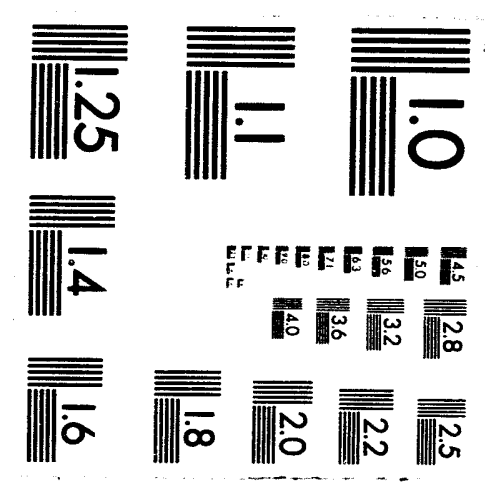



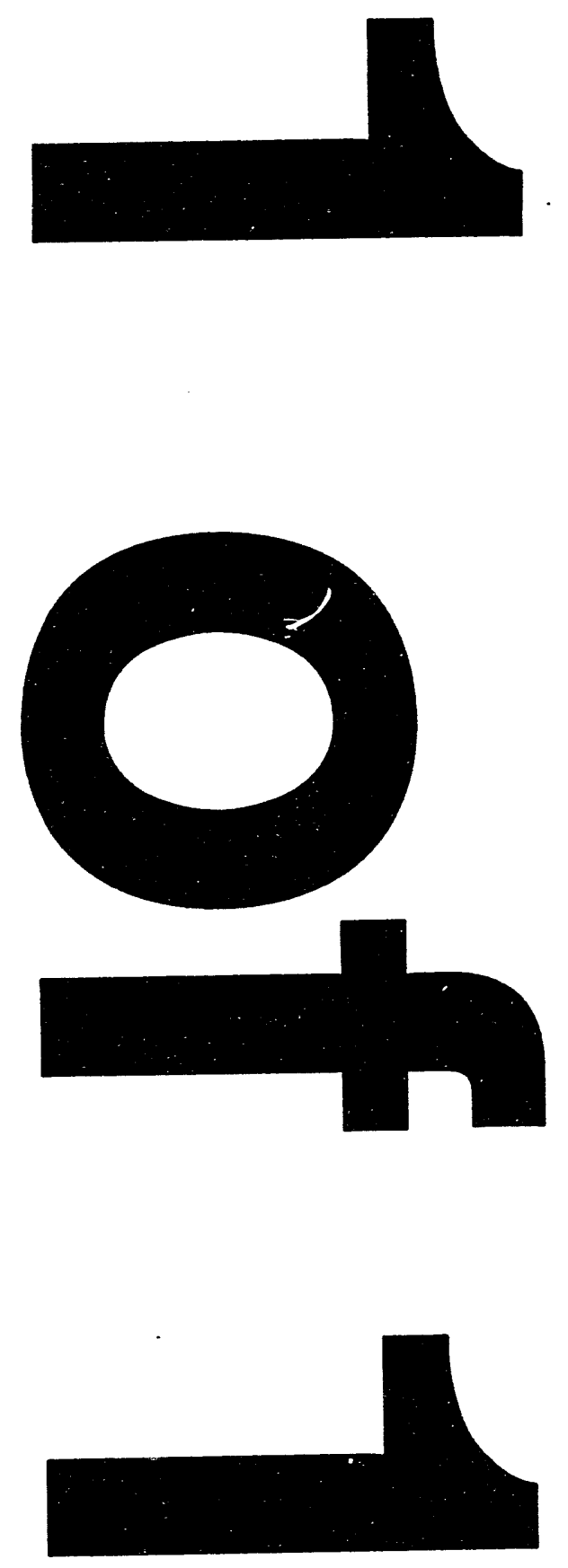


\title{
Corf-940272--1
}

Proceedings of the

Mardi Gras Conference on

"Toward Teraflop Computing and New Grand Challenge Applications"

Baton Rouge, Louisiana

February 10-12, 1994

"The submitted manuscript has been authored by a contractor of the U.S. Goverment under contract No. DE-ACO5-84OR21400. Accordingly, the U.S.

Govemment retains a nonexclusive, royalty-free

Govemment retains a nonexclusive, royalty-free license to publish or reproduce the published form
of this contribution, or allow others to do so, for U.S. Government purposes."

\section{LARGE SCALE ATOMISTIC FIRST PRINCIPLES STUDY OF ADSORPTION AND SURFACE DIFFUSION}

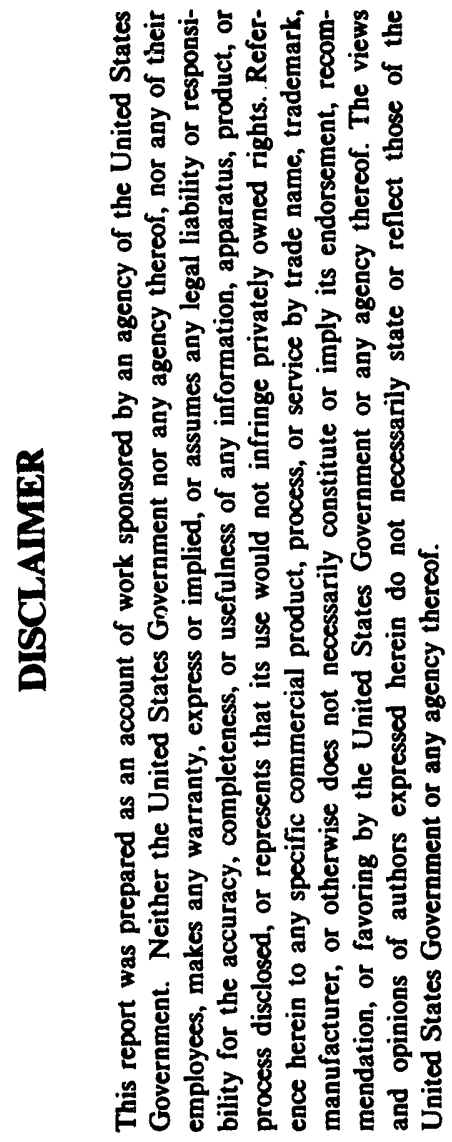

V. Milman, S. J. Pennycook, D. E. Jesson M. C. Payne, I. Stich, and M. H. Lee

\author{
SOLID STATE DIVISION \\ OAK RIDGE NATIONAL LABORATORY \\ Managed by \\ MARTIN MARIETTA ENERGY SYSTEMS, INC. \\ Under \\ Contract No. DE-AC05-84OR21400 \\ With the \\ U. S. DEPARTMENT OF ENERGY \\ OAK RIDGE, TENNESSEE
}

February 1994

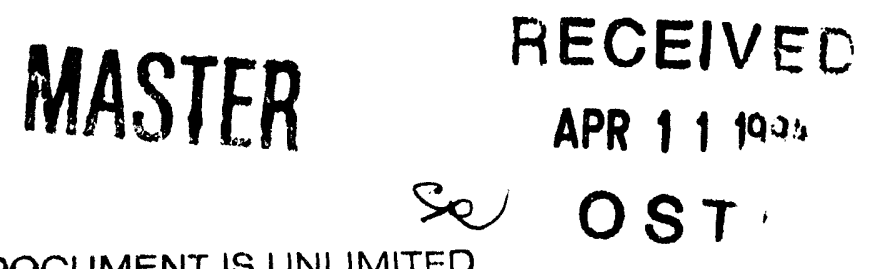




\title{
LARGE SCALE ATOMISTIC FIRST PRINCIPLES STUDY OF ADSORPTION AND SURFACE DIFFUSION
}

\author{
V. MILMAN, S.J. PENNYCOOK, and D.E. JESSON, \\ Solid State Division, Oak Ridge National Laboratory, \\ Oak Ridge, TN 37831 \\ M.C. PAYNE, I. STICH, and M.H.LEE \\ Department of Physics, Cambridge University, \\ Cambridge CB3 OHE, UK
}

\section{INTRODUCTION}

Computer simulation of atomistic processes is an important tool in understanding the properties of condensed matter at a microscopic level. The best way to reproduce the rich variety of experimentally observed effects in the simulation is to use the quantum mechanical description of the ion-electron interactions in a solid. Such calculations although increasingly popular [1] make high demands on the computing power available. The systems of practical interest to surface physics include at least hundreds (or even thousands) of atoms, and their $a b$ initio study inevitably requires parallel supercomputers. In this paper we describe the total energy calculations of the adsorption and migration properties of a single Ge adatom on the $\mathrm{Si}(100)$ surface. This system is typical in complexity for modern large scale simulations and presents a good test case to show the methodology and its application to surface science problems.

The study of fundamental processes in the $\mathrm{Si}-\mathrm{Ge}$ epitaxial growth is of considerable scientific and technological interest [2]. One of the most important questions is the identification of the binding sites for $\mathrm{Ge}$ adatoms on the $\mathrm{Si}(100)$ surface and the determination of the activation energy for surface diffusion. 
Recent STM observations suggest that the behavior of a Ge adatom is essentially similar to that of a $\mathrm{Si}$ adatom. The $\mathrm{Ge}$ islands have the aniso ...py of approximately 1000:1 at $500 \mathrm{~K}$, and the diffusion barrier in the fast direction is estimated to be $0.62 \mathrm{eV}$ [3].

Measurements such as these provide an excellent starting point for theoretical investigation. The only reliable approach for this system is to start from first principles. It is known that empirical potentials describe incorrectly the bonding and diffusion of the $\mathrm{Si}$ adatom on $\mathrm{Si}(100)$ surface (see [4] for a review). None of the empirical potentials available can reproduce even the correct sign of the energy difference between the asymmetric (buckled) and symmetric reconstructions of the $\mathrm{Si}(100)$ surface. One expects that the $\mathrm{Si}-\mathrm{Ge}$ interaction is described by empirical potentials even less accurately.

In the following sections we discuss the parallel implementation of the conjugate gradient technique for $a b$ initio pseudopotential studies of large systems and present our results for $\mathrm{Ge}$ adsorption and diffusion on the $\mathrm{Si}(100)$ surface.

\section{COMPUTATIONAL SCHEME}

We used the pseudopotential total energy approach for the electronic structure calculation. The electronic minimization was performed using band by band algorithm of the conjugate gradient method. The detailed description of this technique is given elsewhere $[1,4]$. A supercell with (100) slab geometry and the $c(4 \times 4)$ surface cell contained 12 layers of $\mathrm{Si}(192$ atoms) and two Ge adatoms with inversion symmetry imposed (Figure 1). The two innermost layers were kept fixed at their bulk positions. A single $\Gamma$-point was used for the Brillouin zone sampling, with an energy cutoff for the plane wave expansion of 10 Ry (about 30000 plane waves). The size of this system makes the computational project feasible only with the use of massively parallel computers. Our calculations were carried out on the Intel computers iPSC/860 and Paragon at the ORNL Center for Computational Science. The details of the implementation of the CETEP pseudopotential code are given in [5], so we just summarize the main points of the parallelization strategy.

The CETEP utilizes the fact that parts of the Hamiltonian are local in either real or reciprocal space, and so the data related to each electronic wavefunction are spread between the processors using space decomposition. We choose the decomposition regions so as to balance the node load and to utilize the structure imposed by the transpose FFT method. The FFT application to wavefunctions is simplified by the fact that some columns would 
be empty due to the difference between the cutoff for wavefunctions and foi the charge density [1]. The main communication overhead occurs in the 3D FFT procedure that is performed as a sequence of 1D transforms with a number of global data exchanges. The nonlocal potential operations are performed in real space taking advantage of the short range of the corresponding operators.

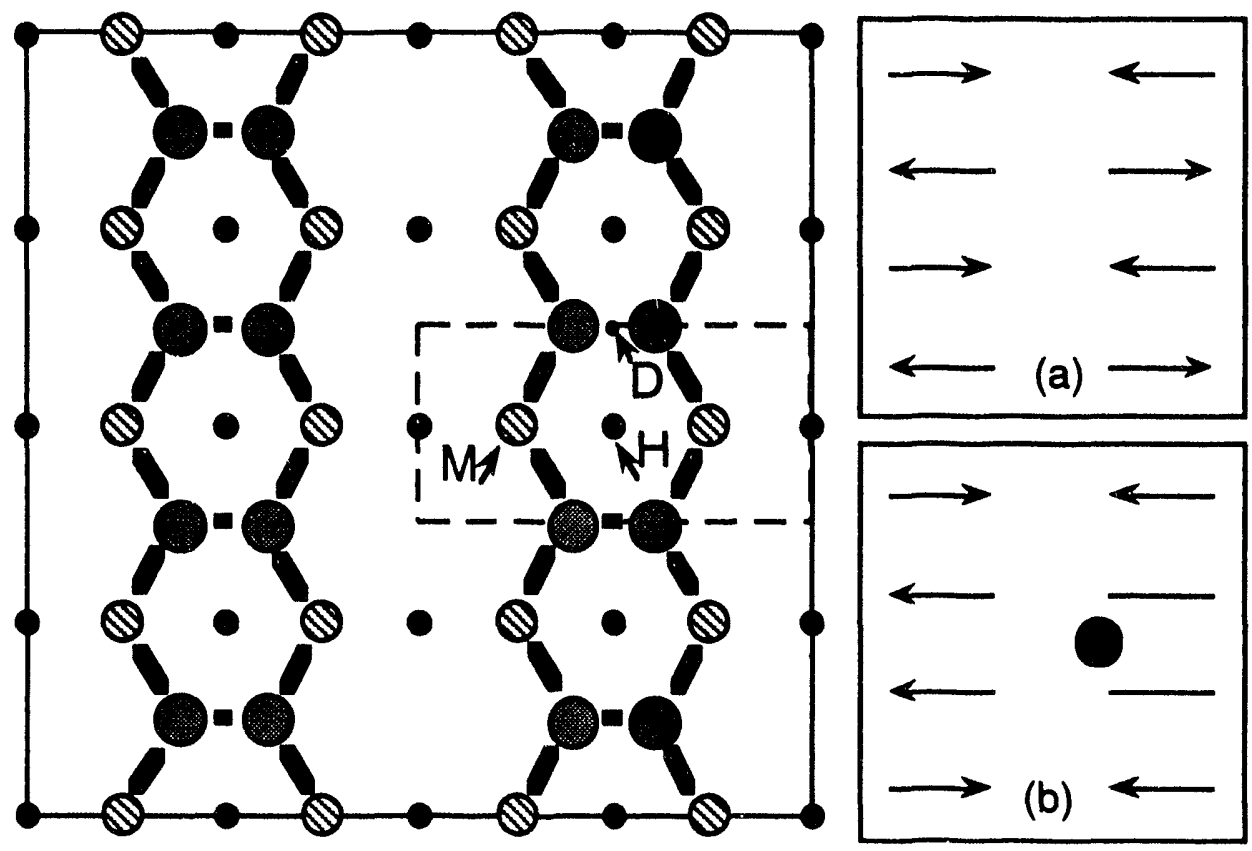

Figure 1. Top view of the three top layers of the $\operatorname{Si}(100)$ surface . The $c(4 \times 4)$ cell is shown by the solid line, $p(2 \times 1)$ by the dashed line. The buckling sequence is represented schematically for the clean surface (a) and in presence of the adatom at site $M(b)$. The arrow points toward a higher atom in the dimer.

The parallel efficiency of the code is difficult to assess on the present Paragon computers with their limited memory per node as one cannot study big system using small number of nodes. On the other hand, an artificial increase of the number of nodes for a small system results in large communication overheads since each node carries too few wavefunction components. The execution time decreased by a factor of 1.6 when using 128 nodes of the Paragon XP/S 35 as compared to 64 nodes. We consider this ratio as a good scaling taking into account that the node load is below the optimum level in case of the 128 node configuration. 
To summarize our experience of using CETEP on massively parallel computers, the main gain is not in the speed-up due to a higher number of processors, but in the ability to approach complex problems that are not tractable by other means.

\section{ENERGY SURFACE FOR THE Ge ADATOM}

The energy surface was mapped out by calculating the total energy for positions $x, y$ of the adatom in the irreducible quarter of the $p(2 \times 1)$ surface cell with the simultaneous total energy minimization with respect to all other ionic coordinates and wave-function expansion coefficients. The local minima were found by allowing the $x, y$ coordinates of the adatom to relax starting from the nearby grid point.

The global minimum is located at point $\mathrm{M}$, and the metastable pedestal site $\mathrm{H}$ is $0.06 \mathrm{eV}$ higher in energy. The diffusion path along the dimer rows is a zig-zag between the point $M$ and the site $D$ which is the saddle point of the trajectory. The barrier height is $0.62 \mathrm{eV}$ in excellent agreement with the experimental estimate [3]. The slow diffusion trajectory has two inequivalent saddle points with the total barrier of $0.95 \mathrm{eV}$. The predicted diffusion anisotropy of $2000: 1$ at $500 \mathrm{~K}$ is in excellent agreement with the STM observations [3]. Thus the STM data are consistent with the $\mathrm{Ge}$ diffusion that takes place on top of the dimers and not in the interdimer trough. The empirical simulations for the $\mathrm{Si}-\mathrm{Ge}$ system are in complete disagreement with $a b$ initio results for the geometry of both adsorption and diffusion.

There is always a possibility of the surface diffusion occuring by an exchange mechanism as opposed to hopping. The barrier for the exchange process is hard to define for a complex surface structure, although its calculation for the $\mathrm{Ge}$ adatom is currently underway. However, this process might be active solely for kinetic reasons, as each exchange act costs $0.11 \mathrm{eV}$ in total energy.

The most controversial issue in the comparison of $a b$ initio and empirical results is the bonding geometry. We find that the bond length between the adatom and the surface atoms is close to the $\mathrm{Si}$ dimer bond length. In equilibrium the $\mathrm{Ge}$ adatom at site $\mathrm{M}$ is bonded to two dimers from the same row, and all bond lengths are about $2.39 \AA$. The distance from the adatom to the second-layer $\mathrm{Si}$ atom is only $2.46 \AA$, but the electron charge density distribution shows that there is no directional bond to this atom. Adsorption at the site $\mathrm{H}$ causes formation of four long bonds of $2.48 \AA$ with the dimer bond stretching to $2.52 \AA$.

The adatom causes maximum strain on dimer bond when it is 
located at the saddle point for the hopping across the trough, or in its vicinity. The distance between the dimer atoms becomes 3.3 $\AA$ which is already closer to the bulk distance of $3.84 \AA$ than to the dimer bond at the clean surface, $2.30 \AA$. No such dimer breaking effect caused by the $\mathrm{Si}$ adatom has been found [6].

\section{EFFECT OF ADATOM ON DIMER BUCKLING}

The interplay between the dimer buckling and the energetics of adsorption and surface diffusion has not been addressed before because of the small energy effect involved and of the prohibitively large size of the corresponding supercell.

During atomic relaxation of the originally symmetric dimers we always observed spontaneous buckling. Its character depends strongly on the adatom position. With the adatom at site $\mathrm{H}$ the dimer row free of adatoms is only slightly perturbed compared to the clean asymmetric $(2 \times 1)$ reconstructed surface. The influence of the adatom is confined then to one dimer row where buckling is entirely suppressed within our supercell.

The picture is qualitatively different in the vicinity of the Ge adatom at the binding site $M$ when both rows are affected (Figure 1). The dimers bonded to the adatom are slightly tilted in the same direction by $0.16 \AA$, and the amplitude grows to $0.43 \AA$ for the next dimers in the same row. A new effect can be seen as a result of the relative proximity of the $\mathrm{Ge}$ adatom to the next dimer row. In that row the two dimers nearest to the adatom has a strong in-phase buckling $(0.62 \AA)$. There is no direct bonding between these dimers and $\mathrm{Ge}$ atom, so this configuration becomes possible due to the second-layer atom displacements. The elastic strain caused by the adatom prevails over the energy gain due to the ferroelectric ordering of buckled dimers and results in the formation of a 1D stacking fault. Since a $\mathrm{Si}$ adatom would produce a qualitatively similar structure, we suggest that the buckling defects observed on $\mathrm{Si}$ (100) [7] might correspond to single Si adatoms. Their imaging gives the only experimental information available on the binding site of the adatom.

\section{CONCLUSIONS}

The first large scale $a b$ initio study of the $\mathrm{Ge}$ bonding and migration on the $\mathrm{Si}(100)$ surface has been performed. The activation barriers for diffusion in fast and slow directions are 0.62 and $0.95 \mathrm{eV}$, respectively, in excellent agreement with the experimental STM results. We identify the localized buckling 
defects observed in STM as being due to the adatom bonded at the equilibrium site implying that the study of single adatoms is experimentally accessible.

\section{ACKNOWLEDGMENTS}

This research was sponsored by the Division of Materials Sciences, U.S. Department of Energy, under contract DE-AC05$840 R 21400$ with Martin Marietta Energy Systems, Inc., by an appointment to the Oak Ridge National Laboratory Postdoctoral Research Program administered by the Oak Ridge Institute for Science and Education. This research is part of the Grand Challenge project on first principles simulation of materials properties, funded under the High-Performance Computing and Communications Initiative.

\section{REFERENCES}

1. M.C.Payne, M.P.Teter, D.C.Allan, T.A.Arias, and J.D.Joannopoulos, "Iterative minimization techniques for $a b$ initio total energy calculations - molecular dynamics and conjugate gradients", Rev. Mod. Phys., Vol. 64, No. 4, 1992, pp. 1045-97.

2. D.E.Jesson, S.J.Pennycook, J.-M.Baribeau, and D.C.Houghton, "Step-driven lateral segregation and long-range ordering during $\mathrm{Si}_{\mathrm{x}} \mathrm{Ge}_{1-\mathrm{x}}$ epitaxial growth", Phys. Rev. Lett., Vol. 68, No. 13,1992 , pp. 2062-5.

3. M.G.Lagally, "An atomic level view of kinetic and thermodynamic influences in the growth of thin films", Jpn. J. Appl. Phys., Vol. 32, No. 3B, 1993, pp.1493-501.

4. V.Milman, D.E.Jesson, S.J.Pennycook, M.C.Payne, M.H.Lee, and I.Stich, " $A b$ Initio Study of the Binding and Diffusion of a $\mathrm{Ge}$ Adatom on the Si(100) Surface", in: "Mechanisms of Thin Film Evolution", eds. S.M.Yalisove, C.V.Thompson, D.J.Eaglesham, MRS 1993 Fall Meeting Proceedings (to be published).

5. L.J.Clarke, I.Stich, and M.C.Payne, "Large scale $a b$ initio total energy calculations on parallel computers", Comp. Phys. Commun., Vol. 72, No. 1, 1992, pp. 14-28.

6. G.Brocks, P.J.Kelly, and R.Car, "Binding and diffusion of a Si adatom on the Si(100) surface", Phys. Rev. Lett., Vol. 66, No. 13, 1991, pp. 1729-32.

7. R.A.Wolkow, "Direct observation of an increase in buckled dimers on $\mathrm{Si}(100)$ at low temperature", Phys. Rev. Lett., Vol. 68, No. 17, 1992, pp. 2636-9. 

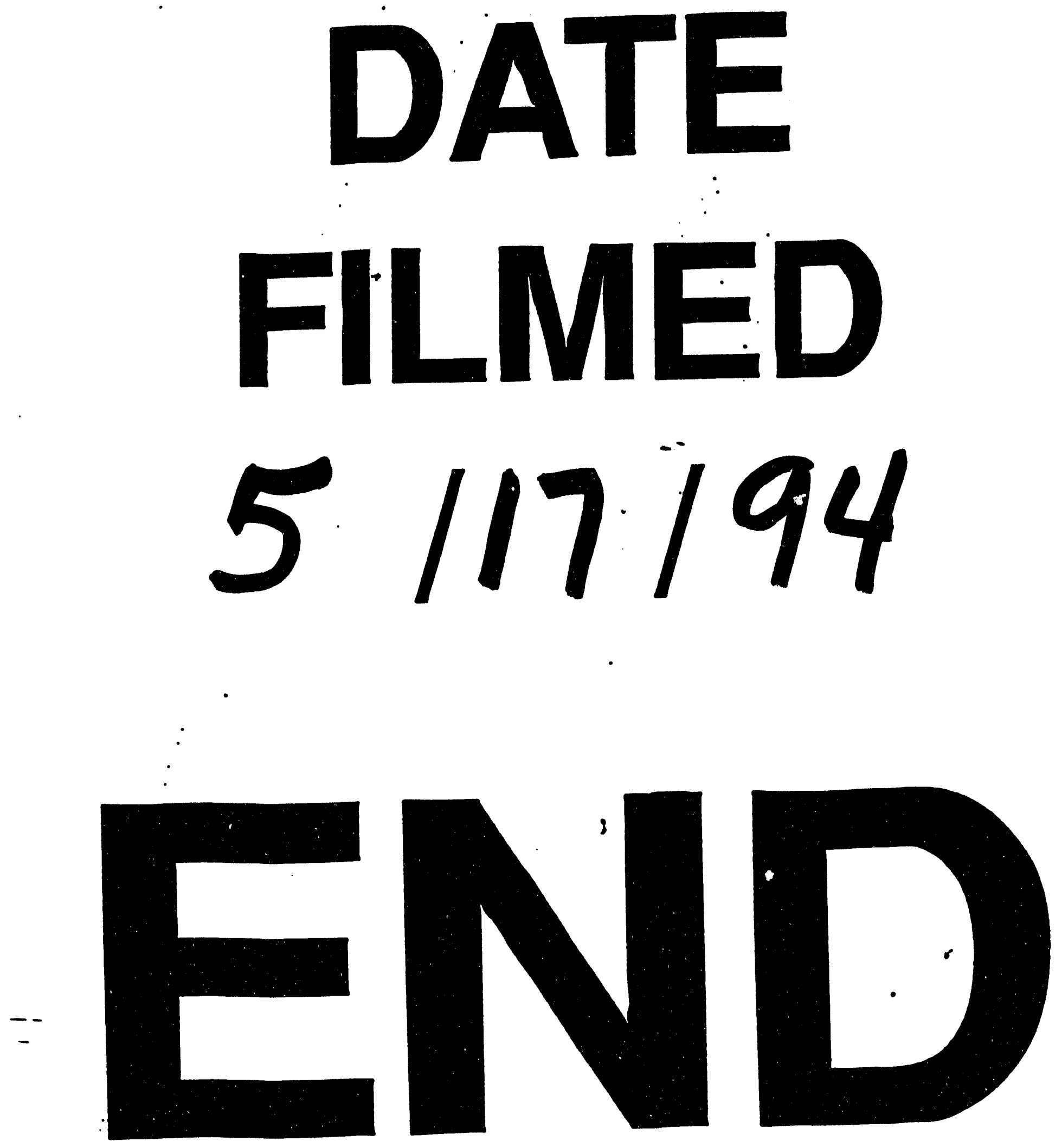
